\title{
Incremental Value of Isolating the Right Inferior Pulmonary Vein During Pulmonary Vein Isolation Procedures in Patients With Paroxysmal Atrial Fibrillation
}

\author{
HAKAN ORAL, AMAN CHUGH, CHRISTOPH SCHARF, BURR HALL, PETER CHEUNG, \\ SRIKAR VEERAREDDY, ERIC GOOD, FRANK PELOSI JR., and FRED MORADY \\ From the Division of Cardiology, University of Michigan, Ann Arbor, Michigan
}

ORAL, H., ET AL.: Incremental Value of Isolating the Right Inferior Pulmonary Vein During Pulmonary Vein Isolation Procedures in Patients With Paroxysmal Atrial Fibrillation. Electrical isolation of the left superior, left inferior, and right superior pulmonary veins (PVs) is often, but not always, effective in eliminating paroxysmal atrial fibrillation $(P A F)$. The incremental clinical value of also isolating the right inferior $P V$ has not been well defined. $P V$ isolation by ostial applications of radiofrequency energy guided by $P V$ potentials was performed in 176 consecutive patients (mean age $52 \pm 11$ years) with PAF. The left superior, left inferior, and right superior PVs were targeted in 106 patients, and all four PVs were targeted 70 patients. Successful isolation was achieved in $96 \%$ of targeted PVs. The mean duration of follow-up was $15 \pm 7$ months. At 1-year follow-up, 58\% of patients in whom three PVs were isolated were free of recurrent PAF in the absence of antiarrhythmic drug therapy, compared to $73 \%$ of patients in whom all four $P V s$ were isolated $(P=0.07)$. There is a trend towards a better outcome when all four PVs are isolated than when only the three major PVs are isolated. Whenever feasible, the right inferior PV should be isolated along with the other three PVs during the first ablation procedure in patients with PAF. (PACE 2004; 27:480-484)

atrial fibrillation, catheter ablation, pulmonary vein

\section{Introduction}

Pulmonary vein (PV) muscle sleeves are capable of generating premature depolarizations and/or bursts of rapid electrical activity that may trigger or drive an episode of atrial fibrillation (AF). ${ }^{1-5}$ This explains why PV isolation may eliminate paroxysmal atrial fibrillation (PAF). ${ }^{6,7}$ The muscle sleeves that surround the left superior, left inferior, and right superior PVs are larger than the muscle sleeves surrounding the right inferior $\mathrm{PV},{ }^{2}$ perhaps explaining why bursts of PV tachycardia are significantly less common in the right inferior PV than in the other three PVs. ${ }^{4}$ Because bursts of tachycardia can be recorded within all three of the major PVs in a majority of episodes of $\mathrm{PAF}^{4}$ the best results with PV isolation have been achieved when the left superior, left inferior, and right superior PVs all are isolated during the first ablation procedure. ${ }^{4,5,7}$

PAF may recur despite isolation of the three major PVs, and it is possible that isolation of the right inferior PV might decrease the recurrence

Supported in part by the Ellen and Robert Thompson Atrial Fibrillation Research Fund, Ann Arbor, MI.

Address for reprints: Hakan Oral, M.D., Cardiology, TC B1 140D, 1500 E. Medical Center Dr., Ann Arbor, MI 48109-0311. Fax: (734) 936-7026; e-mail: oralh@umich.edu

Received July 22, 2003; revised September 24, 2003; accepted October 29, 2003. rate. However, no prior studies have determined the incremental value of including the right inferior PV in PV isolation procedures. Therefore, the purpose of this study was to compare the response to isolation of the three major PVs with and without isolation of the right inferior PV in patients with PAF.

\section{Study Subjects}

\section{Methods}

PV isolation was performed in 176 (143 men, 33 women; mean age $53 \pm 12$, range 19-73 years) patients with symptomatic PAF. The duration of PAF prior to the ablation procedure was $7 \pm$ 7 years, and the mean number of episodes per month was $17 \pm 17$ episodes. A mean of $2 \pm 1$ antiarrhythmic drugs had been ineffective in suppressing the PAF. The mean left ventricular ejection fraction and left atrial diameter were $0.55 \pm$ 0.04 (range 0.35-0.70) and $40 \pm 5 \mathrm{~mm}$ (range 23$57 \mathrm{~mm}$ ), respectively. Structural heart disease was present in $22(13 \%)$ of 176 patients: coronary artery disease in 12, a nonischemic cardiomyopathy in 4 , valvular heart disease in 5 , and congenital heart disease in 1 patient. Seventy of the patients also were subjects of a prior study. ${ }^{7}$

\section{Electrophysiological Procedure}

All patients provided written, informed consent. All catheters were introduced through a 
femoral vein. A quadripolar electrode catheter (EP Technologies, Inc., Mountain View, CA, USA) was positioned in the coronary sinus. After transseptal catheterization, systemic anticoagulation was achieved with intravenous heparin to maintain an activated clotting time of 250 to 350 seconds. Angiograms of the PVs were performed in all patients. Each PV was mapped with a deflectable, circular decapolar catheter (Lasso catheter, Biosense Webster, Diamond Bar, CA, USA). Bipolar and unipolar electrograms were filtered at band-pass settings of $30-500 \mathrm{~Hz}$ and $0.05-200 \mathrm{~Hz}$, respectively, and were recorded digitally (EP MedSystems, Inc., Mt. Arlington, NJ, USA). Pacing was performed from the coronary sinus or left atrial appendage with a stimulator (Model EP-3 Clinical Stimulator, EP MedSystems, Inc.). Radiofrequency energy was delivered via a deflectable, quadripolar 7 Fr catheter with 2-5-2-mm interelectrode spacing and a 4-mm distal electrode with an embedded thermistor (EP Technologies, Inc.).

\section{PV Isolation}

Electrical isolation of the PVs was performed by segmental ostial applications of radiofrequency energy, as described in prior studies. ${ }^{6,7}$ The applications of radiofrequency energy were $30-45$ seconds in duration, with a maximum power of $35 \mathrm{~W}$, and maximum electrode to tissue interface temperature of $52^{\circ} \mathrm{C}$. Elimination of all ostial PV potentials and complete entrance block into the PV were considered indicative of complete electrical isolation.

Only the left superior, left inferior, and right superior PVs were targeted for ablation in 106 patients, and these veins along with the right inferior PV were targeted for isolation in 70 patients. The right inferior PV was included in the PV isolation procedure at the discretion of the primary operator, based primarily on feasibility and procedural duration.

\section{Postablation Care}

After the ablation procedure, all patients underwent continuous monitoring for 24 hours. Heparin was administered intravenously for 24 hours and was followed by low molecular weight heparin for 4 days and warfarin for 1-3 months after the procedure. Patients who had a recurrence of PAF within 4 weeks after the procedure were treated with a class I or III antiarrhythmic drug. Antiarrhythmic drug therapy was discontinued after a patient had been free of PAF for 1-3 months.

\section{Follow-Up}

All patients were seen in the outpatient clinic at 4-6 weeks and every 3-6 months after the ablation procedure. They were instructed to call a nurse practitioner whenever they experienced symptoms, and then were provided with an event recorder to document the cause of their symptoms. During a mean duration of $447 \pm 204$ days, no patient was lost to follow-up.

To look for PV stenosis, computed tomography of the chest with three-dimensional reconstruction of the PVs was performed before the procedure and at 3-6 months after the ablation in $148(84 \%)$ of the 176 patients. $^{8}$

Freedom from recurrent $\mathrm{AF}$ was defined as the absence of symptomatic PAF after discontinuation of all antiarrhythmic drug therapy.

Because episodes of PAF that occur within four weeks after PV isolation may be a transient phenomenon, recurrent PAF that was limited to the first month of follow-up was excluded from the analysis. ${ }^{9}$

\section{Statistical Analysis}

Continuous variables are expressed as mean \pm $1 \mathrm{SD}$ and were compared by Student's $t$-test. Categorical variables were compared by chi-square analysis or with Fisher's exact test. Differences between groups of continuous variables were determined by ANOVA, and post-hoc analyses were performed with the Newman-Keuls test. A KaplanMeier analysis with the log-rank test was used to determine the probability of freedom from recurrent $\mathrm{AF}$ after $\mathrm{PV}$ isolation. A P $<0.05$ was considered statistically significant.

\section{Results \\ Isolation of Three Versus Four PVs}

The clinical characteristics of patients who did and did not undergo isolation of the right inferior PV during the first ablation procedure did not differ significantly (Table I). Among the 106 patients in whom only three PVs were isolated, electrical isolation was complete in $96 \%$ of left superior PVs, 96\% of left inferior PVs, and $97 \%$ of right superior PVs. Among the patients in whom all four PVs were isolated, electrical isolation was complete in $97 \%$ of the left superior PVs, $96 \%$ of the left inferior PVs, 97\% of the right superior PVs, and $100 \%$ of the right inferior PVs. The acute efficacy of PV isolation was similar regardless of whether three or four PVs were targeted for isolation.

The mean duration of radiofrequency energy applications required to achieve complete electrical isolation was $8 \pm 5$ minutes for the left superior PV, $6 \pm 4$ minutes for the right superior PV, $5 \pm$ 4 minutes for the left inferior PV, and $2 \pm 2$ minutes for the right inferior PV $(\mathrm{P}<0.01)$. The duration of radiofrequency energy needed to isolate the right inferior PV was significantly shorter than for the other PVs $(\mathrm{P}<0.01)$. 
Table I.

Characteristics of Patients Who Underwent Isolation of Three or Four PV

\begin{tabular}{lccc}
\hline & $\begin{array}{c}\text { Four PVs } \\
\text { Isolated } \\
\text { (n= 70) }\end{array}$ & $\begin{array}{c}\text { Three PVs } \\
\text { Isolated } \\
\text { (n= 106) }\end{array}$ & P \\
\hline Age (years) & $52 \pm 10$ & $53 \pm 13$ & 0.5 \\
Gender (M/F) & $52 / 18$ & $91 / 15$ & 0.1 \\
Duration of AF (years) & $6 \pm 6$ & $8 \pm 8$ & 0.1 \\
Episodes/mo. of PAF & $19 \pm 19$ & $16 \pm 16$ & 0.3 \\
LA size (mm) & $39 \pm 5$ & $40 \pm 5$ & 0.2 \\
LVEF & $0.56 \pm 0.03$ & $0.55 \pm 0.04$ & 0.1 \\
Heart Disease (Y/N) & $9 / 61$ & $13 / 93$ & 0.9 \\
No. of ineffective & $2 \pm 1$ & $2 \pm 1$ & 0.6 \\
$\quad$ antiarrhythmic drugs & & & \\
\hline
\end{tabular}

Continuous variables expressed as mean $\pm S D$. $L A=$ left atrial; $\mathrm{LVEF}=$ left ventricular ejection fraction; PAF $=$ paroxysmal atrial fibrillation; $\mathrm{PV}=$ pulmonary vein; $\mathrm{RI}=$ right inferior.

\section{Procedure and Fluoroscopy Times}

The mean procedure and fluoroscopy times for isolation of each PV are shown in Table II. The mean additional procedure and floroscopy times for isolation of the right inferior PV were $12 \pm 12$ and $5 \pm 4$ minutes, respectively.

\section{Right Inferior PV, IRAF, and Termination of AF During Ablation}

IRAF was triggered by a burst of PV tachycardia originating in the right inferior PV in three patients. After isolation of the right inferior PV, there were no more episodes of IRAF in these three patients.

PV isolation was performed during an episode of $\mathrm{AF}$ in $68(43 \%)$ of 176 patients. AF terminated during applications of radiofrequency energy in 29

Table II.

Procedural and Fluoroscopy Times

\begin{tabular}{ccccc}
\hline & LS PV & RS PV & LI PV & RI PV \\
\hline $\begin{array}{c}\text { Procedure } \\
\text { Time (min) }\end{array}$ & $34 \pm 21$ & $27 \pm 15$ & $23 \pm 16$ & $12 \pm 12^{*}$ \\
$\begin{array}{c}\text { Fluoroscopy } \\
\text { Time (min) }\end{array}$ & $16 \pm 10$ & $11 \pm 6$ & $11 \pm 8$ & $5 \pm 4^{*}$ \\
\hline
\end{tabular}

Data are shown as mean \pm SD., LS = left superior; RS: right superior; LI: left inferior; RI: right inferior; PV: pulmonary vein. ${ }^{*} \mathrm{P}<0.05$ compared to LS, RS, and LI PVs.

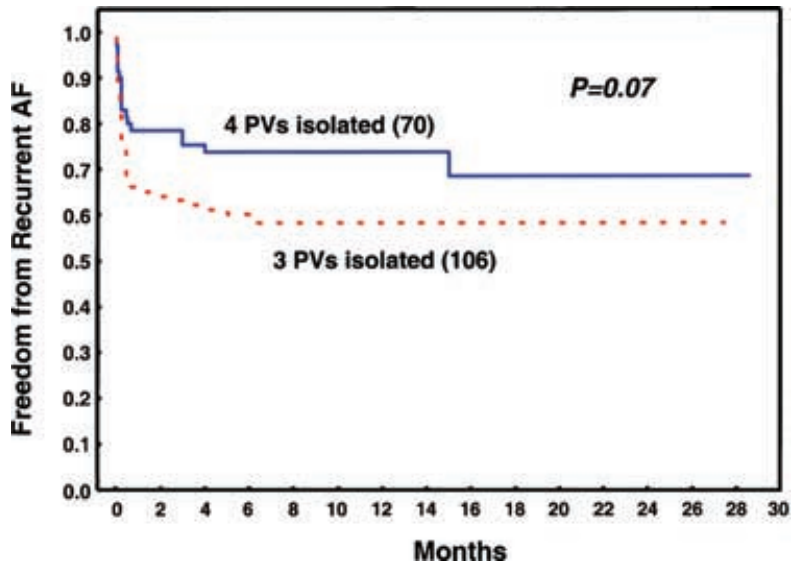

Figure 1. Freedom from recurrent paroxysmal atrial fibrillation after the first ablation procedure in patients who underwent isolation of four pulmonary veins (PVs) (solid line) and three PVs (dashed line) $(P=0.07, \log$ rank test).

(43\%) of 68 patients. Among these 29 patients, sinus rhythm was restored during isolation of the right inferior PV in 2 patients.

\section{Long-Term Freedom From Recurrent AF After Isolation of Three or Four PVs}

Among the 106 patients with PAF who underwent isolation of only the three major PVs, $62 \%$, $58 \%$, and $58 \%$ were free of symptomatic PAF at 6,12 , and 24 months of follow-up, respectively. In comparison, among the 70 patients with PAF who underwent isolation of all four PVs, $75 \%, 73 \%$, and $69 \%$ were free of symptomatic PAF at 6,12 , and 24 months of follow-up, respectively $(\mathrm{P}=0.07$, log-rank test, Fig. 1).

\section{Repeat Ablation Procedures After Isolation of Three or Four PVs}

A repeat ablation procedure for PV isolation by segmental ostial applications of radiofrequency energy was performed in 18 (10\%) of 176 patients with PAF. A repeat ablation procedure was performed in $14(13 \%)$ patients who initially underwent isolation of three PVs, compared to $4(6 \%)$ patients who underwent isolation of all four PVs during the initial ablation procedure $(\mathrm{P}=0.09)$.

In all patients who underwent a repeat ablation procedure, there was recovery of conduction in at least one previously isolated PV.

After the repeat ablation procedure, among 78 patients with PAF in whom all four PVs had been isolated, $80 \%$ and $76 \%$ were free of symptomatic $\mathrm{PAF}$ at 12 and 24 months of follow-up, respectively. In comparison, among 98 patients in whom only the left superior, left inferior, and right superior PVs were isolated, $70 \%$ and $67 \%$ were free of 
symptomatic PAF at 12 and 24 months of followup, respectively $(\mathrm{P}=0.08$, log-rank test).

\section{PV Narrowing}

There was a focal narrowing of $44 \pm 11 \%$ (range 28-61\%) in two left superior and four left inferior PVs in $5(3 \%)$ of the 176 patients. None of the patients developed symptoms attributable to PV stenosis. No PV narrowing or stenosis was observed in any of the right inferior PVs targeted for isolation.

\section{Other Complications}

The incidence of complications other than PV stenosis was 3\% among the patients in whom only three PVs were isolated, compared to $1 \%$ among the patients in whom all four PVs were isolated. The complications consisted of retinal artery embolism $(n=1)$, cardiac tamponade $(n=1)$, and left atrial flutter arising near the left superior $\mathrm{PV}(\mathrm{n}=2)$.

\section{Main Findings}

\section{Discussion}

The results of this study demonstrate that the right inferior PV can be electrically isolated from the left atrium with a total duration of radiofrequency energy that is $<50 \%$ of the duration required for isolation of the other three PVs. In a small percentage of patients with PAF, the right inferior PV can be demonstrated to be a source of IRAF or a source of rapid electrical activity that contributes to maintaining an episode of PAF. Among patients with PAF undergoing their first ablation procedure, isolation of the right inferior PV in addition to the other three PVs is associated with a trend towards a more favorable longterm outcome compared to when only the three other PVs are isolated. Although cannulation of the right inferior PV with a distal ring electrode may be challenging in some patients, in most patients this vein can be successfully isolated with little prolongation in the total PV isolation procedural time.

These results suggest that unless no PV potentials are present at its ostium, whenever feasible, the right inferior PV should be isolated along with the other three PVs during the first ablation procedure in patients with PAF.

\section{Right Inferior PV}

Although inclusion of the right inferior PV in the PV isolation procedure tended to improve the long-term outcome, the degree of improvement at 12 and 24 months of follow-up did not reach statistical significance. This is explained by the observation that arrhythmogenic activity within the right inferior PV was critical in initiating or perpetuating PAF in only a small number of patients in this study. Furthermore, in a prior study, bursts of rapid electrical activity were present during episodes of PAF in $\sim 80 \%$ of the left superior, right superior, and left inferior PVs, compared to only $7 \%$ of right inferior PVs. ${ }^{4}$ The relatively infrequent importance of the right inferior PV in patients with PAF is consistent with prior anatomic studies that have demonstrated that the muscle sleeves around the right inferior PV are less prominent than the muscle sleeves that surround the other PVs. ${ }^{2,10}$

\section{Previous Studies}

In prior studies of PV isolation using conventional or irrigated-tip ablation catheters, ${ }^{6,11}$ efficacy rates of $73 \%$ and $66 \%$ were achieved after mean follow-up intervals of 4 and 8 months. However, $41-49 \%$ of patients in these studies required one or more repeat ablation procedures. In the present study, a success rate of $67-76 \%$ at the 2-year follow-up after isolation of three or four PVs was achieved with only $10 \%$ of patients requiring a repeat procedure. The relatively low redo rate may be attributable to the routine isolation of three to four PVs, regardless of whether or not all of the veins appeared to be arrhythmogenic at the time of the procedure.

\section{Study Limitations}

A limitation of this study is that the decision to isolate the right inferior PV was based on the discretion of the operator, not on a randomized basis. Furthermore, there were no attempts to look for asymptomatic episodes AF.

It is possible that with a larger sample size, a statistically significant difference in efficacy may have been reached. However, this study demonstrated only a trend towards a better outcome when four PVs were isolated than three PVs.

\section{Conclusions}

Although less prevalent than in the other PVs, arrhythmogenic foci capable of generating rapid electrical activity that triggers or drives PAF also may occur within the right inferior PV. Because the right inferior PV usually can be isolated with little added risk or time, and because isolation of four PVs tends to improve the long-term outcome after PV isolation, isolation of all four PVs should be considered during the first ablation procedure in all patients with PAF. However, priority should be given to the other three PVs, and if the right inferior PV is difficult to cannulate or if the time needed to isolate the three major PVs is lengthy, it is appropriate to defer isolation of the right inferior PV until PAF proves to be a recurrent problem. 
ORAL, ET AL.

\section{References}

1. Haissaguerre M, Jais P, Shah DC, et al. Spontaneous initiation of atrial fibrillation by ectopic beats originating in the pulmonary veins. N Engl J Med 1998; 339:659-666.

2. Chen SA, Hsieh MH, Tai CT, et al. Initiation of atrial fibrillation by ectopic beats originating from the pulmonary veins: Electrophysiological characteristics, pharmacological responses, and effects of radiofrequency ablation. Circulation 1999; 100:18791886.

3. Wu TJ, Ong JJ, Chang CM, et al. Pulmonary veins and ligament of marshall as sources of rapid activations in a canine model of sustained atrial fibrillation. Circulation 2001; 103:11571163.

4. Oral $\mathrm{H}$, Ozaydin $\mathrm{M}$, Tada $\mathrm{H}$, et al. Mechanistic significance of intermittent pulmonary vein tachycardia in patients with atrial fibrillation. J Cardiovasc Electrophysiol 2002; 13:645650.

5. Oral H, Knight BP, Ozaydin M, et al. Segmental ostial ablation to isolate the pulmonary veins during atrial fibrillation: Feasibility and mechanistic insights. Circulation 2002; 106:1256-1262.
6. Haissaguerre M, Shah DC, Jais P, et al. Electrophysiological breakthroughs from the left atrium to the pulmonary veins. Circulation 2000; 102:2463-2465.

7. Oral H, Knight BP, Tada H, et al. Pulmonary vein isolation for paroxysmal and persistent atrial fibrillation. Circulation 2002; 105:10771081.

8. Scharf C, Sneider M, Case I, et al. Anatomy of the pulmonary veins in patients with atrial fibrillation and effects of segmental ostial ablation analyzed by computed tomography. J Cardiovasc Electrophysiol 2003; 14:150-155.

9. Oral H, Knight BP, Ozaydin M, et al. Clinical significance of early recurrences of atrial fibrillation after pulmonary vein isolation. Am Coll Cardiol 2002; 40:100-104.

10. Saito T, Waki K, Becker AE. Left atrial myocardial extension onto pulmonary veins in humans: Anatomic observations relevant for atrial arrhythmias. J Cardiovasc Electrophysiol 2000; 11:888-894.

11. Macle L, Jais P, Weerasooriya R, et al. Irrigated-tip catheter ablation of pulmonary veins for treatment of atrial fibrillation. J Cardiovasc Electrophysiol 2002; 13:1067-1073. 